\title{
Jordan Matrix Decomposition
}

\author{
Karol Pąk \\ Institute of Computer Science \\ University of Białystok \\ Poland
}

\begin{abstract}
Summary. In this paper I present the Jordan Matrix Decomposition Theorem which states that an arbitrary square matrix $M$ over an algebraically closed field can be decomposed into the form

$$
M=S J S^{-1}
$$

where $S$ is an invertible matrix and $J$ is a matrix in a Jordan canonical form, i.e. a special type of block diagonal matrix in which each block consists of Jordan blocks (see [13]).
\end{abstract}

MML identifier: MATRIXJ2, version: $\underline{7.9 .01 \quad 4.101 .1015}$

The terminology and notation used here are introduced in the following articles: [11], [2], [3], [12], [34], [7], [10], [8], [4], [28], [33], [30], [18], [6], [14], [15], [36], [23], [37], [35], [9], [29], [32], [31], [5], [19], [24], [22], [17], [1], [21], [20], [16], [25], $[27]$, and $[26]$.

\section{JORDAN BLOCKS}

We follow the rules: $i, j, m, n, k$ denote natural numbers, $K$ denotes a field, and $a, \lambda$ denote elements of $K$.

Let us consider $K, \lambda, n$. The Jordan block of $\lambda$ and $n$ yields a matrix over $K$ and is defined by the conditions (Def. 1).

(Def. 1)(i) len (the Jordan block of $\lambda$ and $n)=n$,

(ii) width (the Jordan block of $\lambda$ and $n$ ) $=n$, and

(iii) for all $i, j$ such that $\langle i, j\rangle \in$ the indices of the Jordan block of $\lambda$ and $n$ holds if $i=j$, then (the Jordan block of $\lambda$ and $n)_{i, j}=\lambda$ and if $i+1=j$, then (the Jordan block of $\lambda$ and $n)_{i, j}=\mathbf{1}_{K}$ and if $i \neq j$ and $i+1 \neq j$, then (the Jordan block of $\lambda$ and $n)_{i, j}=0_{K}$. 
Let us consider $K, \lambda, n$. Then the Jordan block of $\lambda$ and $n$ is an upper triangular matrix over $K$ of dimension $n$.

The following propositions are true:

(1) The diagonal of the Jordan block of $\lambda$ and $n=n \mapsto \lambda$.

(2) Det (the Jordan block of $\lambda$ and $n)=\operatorname{power}_{K}(\lambda, n)$.

(3) The Jordan block of $\lambda$ and $n$ is invertible iff $n=0$ or $\lambda \neq 0_{K}$.

(4) If $i \in \operatorname{Seg} n$ and $i \neq n$, then Line(the Jordan block of $\lambda$ and $n, i)=$ $\lambda \cdot \operatorname{Line}\left(I_{K}^{n \times n}, i\right)+\operatorname{Line}\left(I_{K}^{n \times n}, i+1\right)$.

(5) Line(the Jordan block of $\lambda$ and $n, n)=\lambda \cdot \operatorname{Line}\left(I_{K}^{n \times n}, n\right)$.

(6) Let $F$ be an element of (the carrier of $K)^{n}$ such that $i \in \operatorname{Seg} n$. Then

(i) if $i \neq n$, then Line(the Jordan block of $\lambda$ and $n, i) \cdot F=\lambda \cdot F_{i}+F_{i+1}$, and

(ii) if $i=n$, then Line(the Jordan block of $\lambda$ and $n, i) \cdot F=\lambda \cdot F_{i}$.

(7) Let $F$ be an element of (the carrier of $K)^{n} \operatorname{such}$ that $i \in \operatorname{Seg} n$. Then

(i) $\quad$ if $i=1$, then (the Jordan block of $\lambda$ and $n)_{\square, i} \cdot F=\lambda \cdot F_{i}$, and

(ii) if $i \neq 1$, then (the Jordan block of $\lambda$ and $n)_{\square, i} \cdot F=\lambda \cdot F_{i}+F_{i-1}$.

(8) Suppose $\lambda \neq 0_{K}$. Then there exists a square matrix $M$ over $K$ of dimension $n$ such that

(i) (the Jordan block of $\lambda$ and $n)^{\smile}=M$, and

(ii) for all $i, j$ such that $\langle i, j\rangle \in$ the indices of $M$ holds if $i>j$, then $M_{i, j}=0_{K}$ and if $i \leq j$, then $M_{i, j}=-\operatorname{power}_{K}\left(-\lambda^{-1},\left(j-^{\prime} i\right)+1\right)$.

(9) (The Jordan block of $\lambda$ and $n)+a \cdot I_{K}^{n \times n}=$ the Jordan block of $\lambda+a$ and $n$.

\section{Finite Sequences of Jordan Blocks}

Let us consider $K$ and let $G$ be a finite sequence of elements of $\left((\text { the carrier of } K)^{*}\right)^{*}$. We say that $G$ is Jordan-block-yielding if and only if:

(Def. 2) For every $i$ such that $i \in \operatorname{dom} G$ there exist $\lambda, n$ such that $G(i)=$ the Jordan block of $\lambda$ and $n$.

Let us consider $K$. Observe that there exists a finite sequence of elements of $\left((\text { the carrier of } K)^{*}\right)^{*}$ which is Jordan-block-yielding.

Let us consider $K$. One can verify that every finite sequence of elements of $\left((\text { the carrier of } K)^{*}\right)^{*}$ which is Jordan-block-yielding is also square-matrixyielding.

Let us consider $K$. A finite sequence of Jordan blocks of $K$ is a Jordan-blockyielding finite sequence of elements of $\left((\text { the carrier of } K)^{*}\right)^{*}$.

Let us consider $K, \lambda$. A finite sequence of Jordan blocks of $K$ is said to be a finite sequence of Jordan blocks of $\lambda$ and $K$ if: 
(Def. 3) For every $i$ such that $i \in$ dom it there exists $n$ such that $\operatorname{it}(i)=$ the Jordan block of $\lambda$ and $n$.

Next we state two propositions:

(10) $\emptyset$ is a finite sequence of Jordan blocks of $\lambda$ and $K$.

(11) 〈the Jordan block of $\lambda$ and $n\rangle$ is a finite sequence of Jordan blocks of $\lambda$ and $K$.

Let us consider $K, \lambda$. Observe that there exists a finite sequence of Jordan blocks of $\lambda$ and $K$ which is non-empty.

Let us consider $K$. Note that there exists a finite sequence of Jordan blocks of $K$ which is non-empty.

Next we state the proposition

(12) Let $J$ be a finite sequence of Jordan blocks of $\lambda$ and $K$. Then $J \oplus$ len $J \mapsto$ $a \bullet I_{K}^{\operatorname{Len} J \times \operatorname{Len} J}$ is a finite sequence of Jordan blocks of $\lambda+a$ and $K$.

Let us consider $K$ and let $J_{1}, J_{2}$ be fininte sequences of Jordan blocks of $K$. Then $J_{1} \frown J_{2}$ is a finite sequence of Jordan blocks of $K$.

Let us consider $K$, let $J$ be a finite sequence of Jordan blocks of $K$, and let us consider $n$. Then $J\left\lceil n\right.$ is a finite sequence of Jordan blocks of $K$. Then $J_{\mid n}$ is a finite sequence of Jordan blocks of $K$.

Let us consider $K, \lambda$ and let $J_{1}, J_{2}$ be finite sequences of Jordan blocks of $\lambda$ and $K$. Then $J_{1} \frown J_{2}$ is a finite sequence of Jordan blocks of $\lambda$ and $K$.

Let us consider $K, \lambda$, let $J$ be a finite sequence of Jordan blocks of $\lambda$ and $K$, and let us consider $n$. Then $J\lceil n$ is a finite sequence of Jordan blocks of $\lambda$ and $K$. Then $J_{\mid n}$ is a finite sequence of Jordan blocks of $\lambda$ and $K$.

\section{Nilpotent TRANSFORMATions}

Let $K$ be a double loop structure, let $V$ be a non empty vector space structure over $K$, and let $f$ be a function from $V$ into $V$. We say that $f$ is nilpotent if and only if:

(Def. 4) There exists $n$ such that for every vector $v$ of $V$ holds $f^{n}(v)=0_{V}$.

We now state the proposition

(13) Let $K$ be a double loop structure, $V$ be a non empty vector space structure over $K$, and $f$ be a function from $V$ into $V$. Then $f$ is nilpotent if and only if there exists $n$ such that $f^{n}=\operatorname{ZeroMap}(V, V)$.

Let $K$ be a double loop structure and let $V$ be a non empty vector space structure over $K$. Observe that there exists a function from $V$ into $V$ which is nilpotent.

Let $R$ be a ring and let $V$ be a left module over $R$. Observe that there exists a function from $V$ into $V$ which is nilpotent and linear.

Next we state the proposition 
(14) Let $V$ be a vector space over $K$ and $f$ be a linear transformation from $V$ to $V$. Then $f \uparrow$ ker $f^{n}$ is a nilpotent linear transformation from ker $f^{n}$ to ker $f^{n}$.

Let $K$ be a double loop structure, let $V$ be a non empty vector space structure over $K$, and let $f$ be a nilpotent function from $V$ into $V$. The degree of nilpotence of $f$ yielding a natural number is defined by the conditions (Def. 5).

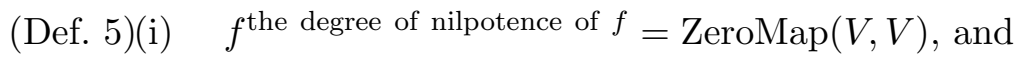

(ii) for every $k$ such that $f^{k}=\operatorname{ZeroMap}(V, V)$ holds the degree of nilpotence of $f \leq k$.

Let $K$ be a double loop structure, let $V$ be a non empty vector space structure over $K$, and let $f$ be a nilpotent function from $V$ into $V$. We introduce $\operatorname{deg} f$ as a synonym of the degree of nilpotence of $f$.

One can prove the following propositions:

(15) Let $K$ be a double loop structure, $V$ be a non empty vector space structure over $K$, and $f$ be a nilpotent function from $V$ into $V$. Then $\operatorname{deg} f=0$ if and only if $\Omega_{V}=\left\{0_{V}\right\}$.

(16) Let $K$ be a double loop structure, $V$ be a non empty vector space structure over $K$, and $f$ be a nilpotent function from $V$ into $V$. Then there exists a vector $v$ of $V$ such that for every $i$ such that $i<\operatorname{deg} f$ holds $f^{i}(v) \neq 0_{V}$.

(17) Let $K$ be a field, $V$ be a vector space over $K, W$ be a subspace of $V$, and $f$ be a nilpotent function from $V$ into $V$. Suppose $f \uparrow W$ is a function from $W$ into $W$. Then $f\lceil W$ is a nilpotent function from $W$ into $W$.

(18) Let $K$ be a field, $V$ be a vector space over $K, W$ be a subspace of $V, f$ be a nilpotent linear transformation from $V$ to $V$, and $f_{1}$ be a nilpotent function from $\operatorname{im}\left(f^{n}\right)$ into $\operatorname{im}\left(f^{n}\right)$. If $f_{1}=f\left\lceil\operatorname{im}\left(f^{n}\right)\right.$ and $n \leq \operatorname{deg} f$, then $\operatorname{deg} f_{1}+n=\operatorname{deg} f$.

For simplicity, we adopt the following convention: $V_{1}, V_{2}$ denote finite dimensional vector spaces over $K, W_{1}, W_{2}$ denote subspaces of $V_{1}, U_{1}, U_{2}$ denote subspaces of $V_{2}, b_{1}$ denotes an ordered basis of $V_{1}, B_{1}$ denotes a finite sequence of elements of $V_{1}, b_{2}$ denotes an ordered basis of $V_{2}, B_{2}$ denotes a finite sequence of elements of $V_{2}, b_{3}$ denotes an ordered basis of $W_{1}, b_{4}$ denotes an ordered basis of $W_{2}, B_{3}$ denotes a finite sequence of elements of $U_{1}$, and $B_{4}$ denotes a finite sequence of elements of $U_{2}$.

Next we state a number of propositions:

(19) Let $M$ be a matrix over $K$ of dimension len $b_{1} \times \operatorname{len} B_{2}, M_{1}$ be a matrix over $K$ of dimension len $b_{3} \times$ len $B_{3}$, and $M_{2}$ be a matrix over $K$ of dimension len $b_{4} \times$ len $B_{4}$ such that $b_{1}=b_{3} \frown b_{4}$ and $B_{2}=B_{3} \frown B_{4}$ and $M=$ the $0_{K}$-block diagonal of $\left\langle M_{1}, M_{2}\right\rangle$ and width $M_{1}=$ len $B_{3}$ and width $M_{2}=$ len $B_{4}$. Then 
(i) if $i \in \operatorname{dom} b_{3}$, then $\left(\operatorname{Mx} 2 \operatorname{Tran}\left(M, b_{1}, B_{2}\right)\right)\left(\left(b_{1}\right)_{i}\right)=$ $\left(\operatorname{Mx} 2 \operatorname{Tran}\left(M_{1}, b_{3}, B_{3}\right)\right)\left(\left(b_{3}\right)_{i}\right)$, and

(ii) if $i \in \operatorname{dom} b_{4}$, then $\left(\operatorname{Mx} 2 \operatorname{Tran}\left(M, b_{1}, B_{2}\right)\right)\left(\left(b_{1}\right)_{i+\operatorname{len} b_{3}}\right)=$ $\left(\mathrm{Mx} 2 \operatorname{Tran}\left(M_{2}, b_{4}, B_{4}\right)\right)\left(\left(b_{4}\right)_{i}\right)$.

(20) Let $M$ be a matrix over $K$ of dimension len $b_{1} \times \operatorname{len} B_{2}$ and $F$ be a finite sequence of matrices over $K$. Suppose $M=$ the $0_{K}$-block diagonal of $F$. Let given $i, m$. Suppose $i \in \operatorname{dom} b_{1}$ and $m=\min (\operatorname{Len} F, i)$. Then $\left(\operatorname{Mx} 2 \operatorname{Tran}\left(M, b_{1}, B_{2}\right)\right)\left(\left(b_{1}\right)_{i}\right)=\sum \operatorname{lmlt}\left(\operatorname{Line}\left(F(m), i-^{\prime} \sum \operatorname{Len}\left(F \uparrow\left(m-{ }^{\prime}\right.\right.\right.\right.$ $1))),\left(B_{2}\left\lceil\sum \operatorname{Width}(F\lceil m))_{\mid \sum \operatorname{Width}\left(F\left\lceil\left(m-^{\prime} 1\right)\right)\right.}\right)\right.$ and $\operatorname{len}\left(\left(B_{2} \uparrow \sum \operatorname{Width}(F\lceil m))_{\mid \sum \operatorname{Width}\left(F\left\lceil\left(m-^{\prime} 1\right)\right)\right.}\right)=\right.$ width $F(m)$.

(21) If len $B_{1} \in \operatorname{dom} B_{1}$, then $\sum \operatorname{lmlt}($ Line(the Jordan block of $\lambda$ and len $B_{1}$, len $\left.\left.B_{1}\right), B_{1}\right)=\lambda \cdot\left(B_{1}\right)_{\operatorname{len} B_{1}}$.

(22) If $i \in \operatorname{dom} B_{1}$ and $i \neq \operatorname{len} B_{1}$, then $\sum \operatorname{lmlt}($ Line(the Jordan block of $\lambda$ and len $\left.\left.B_{1}, i\right), B_{1}\right)=\lambda \cdot\left(B_{1}\right)_{i}+\left(B_{1}\right)_{i+1}$.

(23) Let $M$ be a matrix over $K$ of dimension len $b_{1} \times$ len $B_{2}$. Suppose $M=$ the Jordan block of $\lambda$ and $n$. Let given $i$ such that $i \in \operatorname{dom} b_{1}$. Then

(i) if $i=\operatorname{len} b_{1}$, then $\left(\operatorname{Mx} 2 \operatorname{Tran}\left(M, b_{1}, B_{2}\right)\right)\left(\left(b_{1}\right)_{i}\right)=\lambda \cdot\left(B_{2}\right)_{i}$, and

(ii) if $i \neq$ len $b_{1}$, then $\left(\operatorname{Mx} 2 \operatorname{Tran}\left(M, b_{1}, B_{2}\right)\right)\left(\left(b_{1}\right)_{i}\right)=\lambda \cdot\left(B_{2}\right)_{i}+\left(B_{2}\right)_{i+1}$.

(24) Let $J$ be a finite sequence of Jordan blocks of $\lambda$ and $K$ and $M$ be a matrix over $K$ of dimension len $b_{1} \times$ len $B_{2}$. Suppose $M=$ the $0_{K}$-block diagonal of $J$. Let given $i, m$ such that $i \in \operatorname{dom} b_{1}$ and $m=\min (\operatorname{Len} J, i)$. Then

(i) if $i=\sum \operatorname{Len}\left(J\lceil m)\right.$, then $\left(\operatorname{Mx} 2 \operatorname{Tran}\left(M, b_{1}, B_{2}\right)\right)\left(\left(b_{1}\right)_{i}\right)=\lambda \cdot\left(B_{2}\right)_{i}$, and

(ii) if $i \neq \sum \operatorname{Len}\left(J\lceil m)\right.$, then $\left(\operatorname{Mx} 2 \operatorname{Tran}\left(M, b_{1}, B_{2}\right)\right)\left(\left(b_{1}\right)_{i}\right)=\lambda \cdot\left(B_{2}\right)_{i}+$ $\left(B_{2}\right)_{i+1}$.

(25) Let $J$ be a finite sequence of Jordan blocks of $0_{K}$ and $K$ and $M$ be a matrix over $K$ of dimension len $b_{1} \times$ len $b_{1}$. Suppose $M=$ the $0_{K}$-block diagonal of $J$. Let given $m$. If for every $i$ such that $i \in \operatorname{dom} J$ holds len $J(i) \leq m$, then $\left(\operatorname{Mx} 2 \operatorname{Tran}\left(M, b_{1}, b_{1}\right)\right)^{m}=\operatorname{ZeroMap}\left(V_{1}, V_{1}\right)$.

(26) Let $J$ be a finite sequence of Jordan blocks of $\lambda$ and $K$ and $M$ be a matrix over $K$ of dimension len $b_{1} \times$ len $b_{1}$. Suppose $M=$ the $0_{K}$-block diagonal of $J$. Then $\operatorname{Mx} 2 \operatorname{Tran}\left(M, b_{1}, b_{1}\right)$ is nilpotent if and only if len $b_{1}=0$ or $\lambda=0_{K}$.

(27) Let $J$ be a finite sequence of Jordan blocks of $0_{K}$ and $K$ and $M$ be a matrix over $K$ of dimension len $b_{1} \times$ len $b_{1}$. Suppose $M=$ the $0_{K}$-block diagonal of $J$ and len $b_{1}>0$. Let $F$ be a nilpotent function from $V_{1}$ into $V_{1}$. Suppose $F=\operatorname{Mx} 2 \operatorname{Tran}\left(M, b_{1}, b_{1}\right)$. Then there exists $i$ such that $i \in \operatorname{dom} J$ and len $J(i)=\operatorname{deg} F$ and for every $i$ such that $i \in \operatorname{dom} J$ holds len $J(i) \leq \operatorname{deg} F$.

(28) Let given $V_{1}, V_{2}, b_{1}, b_{2}, \lambda$. Suppose len $b_{1}=\operatorname{len} b_{2}$. Let $F$ be a linear 
transformation from $V_{1}$ to $V_{2}$. Suppose that for every $i$ such that $i \in \operatorname{dom} b_{1}$ holds $F\left(\left(b_{1}\right)_{i}\right)=\lambda \cdot\left(b_{2}\right)_{i}$ or $i+1 \in \operatorname{dom} b_{1}$ and $F\left(\left(b_{1}\right)_{i}\right)=\lambda \cdot\left(b_{2}\right)_{i}+\left(b_{2}\right)_{i+1}$. Then there exists a non-empty finite sequence $J$ of Jordan blocks of $\lambda$ and $K$ such that $\operatorname{AutMt}\left(F, b_{1}, b_{2}\right)=$ the $0_{K}$-block diagonal of $J$.

(29) Let $V_{1}$ be a finite dimensional vector space over $K$ and $F$ be a nilpotent linear transformation from $V_{1}$ to $V_{1}$. Then there exists a non-empty finite sequence $J$ of Jordan blocks of $0_{K}$ and $K$ and there exists an ordered basis $b_{1}$ of $V_{1}$ such that $\operatorname{AutMt}\left(F, b_{1}, b_{1}\right)=$ the $0_{K}$-block diagonal of $J$.

(30) Let $V$ be a vector space over $K, F$ be a linear transformation from $V$ to $V, V_{1}$ be a finite dimensional subspace of $V$, and $F_{1}$ be a linear transformation from $V_{1}$ to $V_{1}$. Suppose $V_{1}=\operatorname{ker}\left(F+(-\lambda) \cdot \operatorname{id}_{V}\right)^{n}$ and $F\left\lceil V_{1}=F_{1}\right.$. Then there exists a non-empty finite sequence $J$ of Jordan blocks of $\lambda$ and $K$ and there exists an ordered basis $b_{1}$ of $V_{1}$ such that $\operatorname{AutMt}\left(F_{1}, b_{1}, b_{1}\right)=$ the $0_{K}$-block diagonal of $J$.

\section{The Main Theorem}

The following two propositions are true:

(31) Let $K$ be an algebraic-closed field, $V$ be a non trivial finite dimensional vector space over $K$, and $F$ be a linear transformation from $V$ to $V$. Then there exists a non-empty finite sequence $J$ of Jordan blocks of $K$ and there exists an ordered basis $b_{1}$ of $V$ such that

(i) $\operatorname{AutMt}\left(F, b_{1}, b_{1}\right)=$ the $0_{K}$-block diagonal of $J$, and

(ii) for every scalar $\lambda$ of $K$ holds $\lambda$ is an eigenvalue of $F$ iff there exists $i$ such that $i \in \operatorname{dom} J$ and $J(i)=$ the Jordan block of $\lambda$ and len $J(i)$.

(32) Let $K$ be an algebraic-closed field and $M$ be a square matrix over $K$ of dimension $n$. Then there exists a non-empty finite sequence $J$ of Jordan blocks of $K$ and there exists a square matrix $P$ over $K$ of dimension $n$ such that $\sum$ Len $J=n$ and $P$ is invertible and $M=P$. the $0_{K}$-block diagonal of $J \cdot P^{\smile}$.

\section{REFERENCES}

[1] Jesse Alama. The rank+nullity theorem. Formalized Mathematics, 15(3):137-142, 2007.

[2] Grzegorz Bancerek. Cardinal numbers. Formalized Mathematics, 1(2):377-382, 1990.

[3] Grzegorz Bancerek. The fundamental properties of natural numbers. Formalized Mathematics, 1(1):41-46, 1990.

[4] Grzegorz Bancerek and Krzysztof Hryniewiecki. Segments of natural numbers and finite sequences. Formalized Mathematics, 1(1):107-114, 1990.

[5] Czesław Byliński. Binary operations applied to finite sequences. Formalized Mathematics, 1(4):643-649, 1990.

[6] Czesław Byliński. Finite sequences and tuples of elements of a non-empty sets. Formalized Mathematics, 1(3):529-536, 1990.

[7] Czesław Byliński. Functions and their basic properties. Formalized Mathematics, 1(1):5565, 1990. 
[8] Czesław Byliński. Functions from a set to a set. Formalized Mathematics, 1(1):153-164, 1990.

[9] Czesław Byliński. The modification of a function by a function and the iteration of the composition of a function. Formalized Mathematics, 1(3):521-527, 1990.

[10] Czesław Byliński. Partial functions. Formalized Mathematics, 1(2):357-367, 1990.

[11] Czesław Byliński. Some basic properties of sets. Formalized Mathematics, 1(1):47-53, 1990.

[12] Agata Darmochwał. Finite sets. Formalized Mathematics, 1(1):165-167, 1990.

[13] Gene H. Golub and J. H. Wilkinson. Ill-conditioned eigensystems and the computation of the Jordan normal form. SIAM Review, vol. 18, nr. 4, pp. 5788211;619, 1976.

[14] Katarzyna Jankowska. Matrices. Abelian group of matrices. Formalized Mathematics, 2(4):475-480, 1991.

[15] Katarzyna Jankowska. Transpose matrices and groups of permutations. Formalized Mathematics, 2(5):711-717, 1991.

[16] Andrzej Kondracki. The Chinese Remainder Theorem. Formalized Mathematics, 6(4):573-577, 1997.

[17] Jarosław Kotowicz. Functions and finite sequences of real numbers. Formalized Mathematics, 3(2):275-278, 1992.

[18] Eugeniusz Kusak, Wojciech Leończuk, and Michał Muzalewski. Abelian groups, fields and vector spaces. Formalized Mathematics, 1(2):335-342, 1990.

[19] Robert Milewski. Associated matrix of linear map. Formalized Mathematics, 5(3):339345,1996

[20] Robert Milewski. Fundamental theorem of algebra. Formalized Mathematics, 9(3):461$470,2001$.

[21] Michał Muzalewski. Categories of groups. Formalized Mathematics, 2(4):563-571, 1991.

[22] Michał Muzalewski. Rings and modules - part II. Formalized Mathematics, 2(4):579-585, 1991.

[23] Takaya Nishiyama and Yasuho Mizuhara. Binary arithmetics. Formalized Mathematics, 4(1):83-86, 1993.

[24] Karol Pạk and Andrzej Trybulec. Laplace expansion. Formalized Mathematics, 15(3):143$150,2007$.

[25] Karol Pąk. Block diagonal matrices. Formalized Mathematics, 16(3):259-267, 2008.

[26] Karol Pąk. Eigenvalues of a linear transformation. Formalized Mathematics, 16(4):289295, 2008.

[27] Karol Pąk. Linear map of matrices. Formalized Mathematics, 16(3):269-275, 2008.

[28] Andrzej Trybulec. Binary operations applied to functions. Formalized Mathematics, 1(2):329-334, 1990.

[29] Andrzej Trybulec. Domains and their Cartesian products. Formalized Mathematics, 1(1):115-122, 1990.

[30] Wojciech A. Trybulec. Groups. Formalized Mathematics, 1(5):821-827, 1990.

[31] Wojciech A. Trybulec. Linear combinations in vector space. Formalized Mathematics, $1(5): 877-882,1990$.

[32] Wojciech A. Trybulec. Subspaces and cosets of subspaces in vector space. Formalized Mathematics, 1(5):865-870, 1990.

[33] Wojciech A. Trybulec. Vectors in real linear space. Formalized Mathematics, 1(2):291-296, 1990.

[34] Edmund Woronowicz. Relations and their basic properties. Formalized Mathematics, 1(1):73-83, 1990.

[35] Xiaopeng Yue, Xiquan Liang, and Zhongpin Sun. Some properties of some special matrices. Formalized Mathematics, 13(4):541-547, 2005.

[36] Katarzyna Zawadzka. The sum and product of finite sequences of elements of a field. Formalized Mathematics, 3(2):205-211, 1992.

[37] Katarzyna Zawadzka. The product and the determinant of matrices with entries in a field. Formalized Mathematics, 4(1):1-8, 1993.

Received July 11, 2008 